\title{
State Feedback Stabilization for Neutral-Type Neural Networks with Time-Varying Discrete and Unbounded Distributed Delays
}

\author{
Yantao Wang, Xue Lin, and Xian Zhang \\ School of Mathematical Science, Heilongjiang University, Harbin 150080, China \\ Correspondence should be addressed to Xian Zhang, xianzhang@ieee.org
}

Received 19 December 2011; Revised 27 March 2012; Accepted 21 April 2012

Academic Editor: Onur Toker

Copyright (c) 2012 Yantao Wang et al. This is an open access article distributed under the Creative Commons Attribution License, which permits unrestricted use, distribution, and reproduction in any medium, provided the original work is properly cited.

\begin{abstract}
The problem of stabilization for a class of neutral-type neural networks with discrete and unbounded distributed delays is investigated. By introducing an appropriate Lyapunov-Krasovskii functional and using Jensen inequality technique to deal with its derivative, delay-range-dependent and rate-dependent stabilization criteria are presented in the form of LMIs with nonlinear constraints. In order to solve the nonlinear problem, a cone complementarity linearization (CCL) algorithm is offered. In addition, several numerical examples are provided to illustrate the applicability of the proposed approach.
\end{abstract}

\section{Introduction}

In 1943, McCulloch and Pitts [1] proposed the concept of artificial neurons and proved that a single neuron can perform logic functions. This is usually viewed as the beginning of era of artificial neural networks, which is called neural networks for short. Recently, neural networks have received considerable attention due to their wide applications in solving some optimization problems, pattern recognition, image processing, and signal processing. Since time delay is frequently a source of instability and/or oscillation of many practical systems, several approaches have been proposed for analysis and synthesis of delayed neural networks (see [2-8] and the references therein).

A neutral-type time-delay system contains delays in the state and its derivatives. Such system can be found in population ecology, lossless transmission lines, heat exchangers, and so forth. Because of its wider applications, the stability for the class of neutral-type systems has received considerable attention in the last several decades (see, e.g., [9-13]). Corresponding to the class of neutral-type timedelay systems, we will also get a class of neutral-type neural networks. Stability criteria for neutral-type neural networks have been proposed in $[3,14-21]$ by constructing appropriate Lyapunov-Krasovskii functional and applying the linear matrix inequality (LMI) approach. Recently, the stability conditions for the neutral-type neural networks with discrete and unbounded distributed delays were provided in [1721]. Specifically, Rakkiyappan and Balasubramaniam [21] studied the global asymptotic stability for a class of neutraltype neural networks with two unbounded distributed delays by the so-called LMI technique. Lu [22] designed a state feedback controller stabilizing the neutral-type neural networks with discrete and bounded distributed delays. However, there are no results about the stabilization for neutral-type neural networks with unbounded distributed $\operatorname{delay}(\mathrm{s})$, this motivates our research.

This paper deals with the stabilization problem of a class of neutral-type neural networks with time-varying discrete and unbounded distributed delays. New sufficient conditions for the existence of the state feedback controller are proposed by introducing a Lyapunov-Krasovskii functional and using Jensen inequality technique to deal with its derivative. Since these sufficient conditions are presented in the form of LMIs with nonlinear constraints, the so-called CCL technique is employed to deal with the nonlinear constraints. This allows us to obtain a state feedback gain by LMI Control Toolbox of MATLAB. Several numerical examples are given to illustrate the effectiveness of the proposed approach. 


\section{Problem Formulation}

Consider the class of neutral-type neural networks with time-varying discrete and unbounded distributed delays described by the integro differential equation:

$$
\begin{gathered}
\dot{x}_{i}(t)=-a_{i} x_{i}(t)+\sum_{j=1}^{n} b_{i j} f_{j}\left(x_{j}(t)\right)+\sum_{j=1}^{n} c_{i j} f_{j}\left(x_{j}(t-\tau(t))\right) \\
+\sum_{j=1}^{n} e_{i j} \int_{-\infty}^{t} z_{j}(t-s) f_{j}\left(x_{j}(s)\right) \mathrm{d} s+\sum_{j=1}^{n} d_{i j} \dot{x}_{j}(t-h(t)) \\
+\sum_{j=1}^{n} f_{i j} \int_{-\infty}^{t} z_{j}(t-s) \dot{x}_{j}(s) \mathrm{d} s+\ell_{i}+I_{i}(t), \\
x_{i}(\theta)=\phi_{i}(\theta), \quad \forall \theta \in(-\infty, 0],
\end{gathered}
$$

where $i=1,2, \ldots, n, x_{i}(t)$ is the state variable of the $i$ th neuron, $a_{i}>0$ is a constant, $b_{i j}, c_{i j}, d_{i j}, e_{i j}$, and $f_{i j}$ are connection weight coefficients of the neurons, $f_{j}$ is the neuron activation function, $\ell_{i}$ is the external bias vector element, $I_{i}(t)$ is the external control input vector, $\phi_{i}(\theta)$ denotes the initial condition, $\tau(t)$ and $h(t)$ are time-varying delays satisfying

$$
\begin{array}{ll}
0 \leq \tau_{1} \leq \tau(t) \leq \tau_{2}, & \dot{\tau}(t) \leq \tau_{3}, \\
0 \leq h_{1} \leq h(t) \leq h_{2}, & \dot{h}(t) \leq h_{3},
\end{array}
$$

and $z_{j}(t)$ is a real value nonnegative continuous function on $[0,+\infty)$ and satisfies

$$
\int_{0}^{\infty} z_{j}(s) \mathrm{d} s=1
$$

When $I_{i}(t) \equiv 0$ for all $i$, the global asymptotic stability of the system (1) has been considered in [21]. Here, the control input item $I_{i}(t)$ in (1) is borrowed from [22].

Throughout this paper, we assume that the neuron activation functions $f_{j}(j=1,2, \ldots, n)$ satisfy the following condition:

$$
\left|f_{j}\left(\zeta_{1}\right)-f_{j}\left(\zeta_{2}\right)\right| \leq L_{j}\left|\zeta_{1}-\zeta_{2}\right| \quad \forall \zeta_{1}, \zeta_{2} \in \mathbb{R}, \zeta_{1} \neq \zeta_{2},
$$

where $L_{j}(j=1,2, \ldots, n)$ are known positive constants.

Let $x^{*}=\left(x_{1}^{*}, x_{2}^{*}, \ldots, x_{n}^{*}\right)$ be an equilibrium point of the system (1). Set $y_{i}=x_{i}-x_{i}^{*}, i=1, \ldots, n$. Then the system (1) can be written as

$$
\begin{aligned}
& \dot{y}(t)=-A y(t)+B g(y(t)) \\
&+C g(y(t-\tau(t)))+D \dot{y}(t-h(t)) \\
&+E \int_{-\infty}^{t} Z(t-s) g(y(s)) \mathrm{d} s \\
&+F \int_{-\infty}^{t} Z(t-s) \dot{y}(s) \mathrm{d} s+I(t), \\
& y(\theta)=\phi(\theta), \quad \forall \theta \in(-\infty, 0],
\end{aligned}
$$

where $y(t)=\left[y_{1}(t) y_{2}(t) \cdots y_{n}(t)\right]^{T}, A=\operatorname{diag}\left(a_{1}, a_{2}, \ldots\right.$, $\left.a_{n}\right), B=\left[b_{i j}\right], C=\left[c_{i j}\right], D=\left[d_{i j}\right], E=\left[e_{i j}\right], F=\left[f_{i j}\right]$, $Z(t)=\operatorname{diag}\left(z_{1}(t), z_{2}(t), \ldots, z_{n}(t)\right), I(t)=\left[I_{1}(t) \cdots I_{n}(t)\right]^{T}$, $g(y(t))=\left[g_{1}\left(y_{1}(t)\right) \mathrm{g}_{2}\left(y_{2}(t)\right) \cdots g_{n}\left(y_{n}(t)\right)\right]^{T}$ with $g_{j}\left(y_{j}(t)\right)$ $=f_{j}\left(y_{j}(t)+x_{j}^{*}\right)-f_{j}\left(x_{j}^{*}\right)$, and $\phi(\theta)=\left[\phi_{1}(\theta)-x_{1}^{*} \cdots \phi_{n}(\theta)-\right.$ $\left.x_{n}^{*}\right]$. From (5), we have $g_{j}^{2}(\zeta) \leq L_{j}^{2} \zeta^{2}, j=1,2, \ldots, n$, for all $\zeta \in \mathbb{R}$.

The main purpose of this paper is to develop a delayrange-dependent and rate-dependent condition for the existence of a state feedback controller

$$
I(t)=K y(t)
$$

which stabilizes the neutral-type neural network (6) with time-varying discrete and unbounded distributed delays.

A delay-range-dependent and rate-dependent criterion stabilizing (6) is obtained based on a Lyapunov-Krasovskii functional approach accompanied with a CCL technique, a delay-range-dependent and rate-dependent criterion stabilizing (6) is obtained. Set $y_{t}(\theta)=y(t+\theta), \theta \in$ $(-\infty, 0]$. Assume that there exists a scalar $V>0$ such that $\int_{-\infty}^{0}\left\|y_{t}(s)\right\|^{2} \mathrm{~d} s \leq V$ and $\int_{-\infty}^{0}\left\|\dot{y}_{t}(s)\right\|^{2} \mathrm{~d} s \leq V$. This guarantees that the so-called Lyapunov-Krasovskii functional theory can be applied to the considered stabilization problem.

\section{Main Results}

In this section, we will investigate a delay-range-dependent and rate-dependent stabilization criterion for the neutraltype neural network (6). This requires the following several lemmas.

Lemma 1 (Jensen inequality [23]). Given a real symmetry positive-definite matrix $P \in \mathbb{R}^{n \times n}$, a pair of scalars $a$ and $b$ satisfying $b \geq a \geq 0$. If a vector-valued function $x:[a, b] \rightarrow$ $\mathbb{R}^{n}$ is derivable on $[a, b]$, then

$$
(b-a) \int_{a}^{b} \dot{x}^{T}(w) P \dot{x}(w) \mathrm{d} w \geq[x(b)-x(a)]^{T} P[x(b)-x(a)] .
$$

Lemma 2 (Schur complementary lemma [24]). For a given matrix $S:=\left[\begin{array}{ll}S_{11} & S_{12} \\ S_{12}^{T} & S_{22}\end{array}\right]$ with $S_{11}=S_{11}^{T}$ and $S_{22}=S_{22}^{T}$, the following conditions are equivalent.

(i) $S<0$.

(ii) $S_{22}<0$ and $S_{11}-S_{12} S_{22}^{-1} S_{12}^{T}<0$.

(iii) $S_{11}<0$ and $S_{22}-S_{12}^{T} S_{11}^{-1} S_{12}<0$.

Lemma 3 (Cauchy-Schwarz inequality [25]). If the functions $f$ and $g$ are integral on $[a, b]$, then

$$
\left(\int_{a}^{b} f(s) g(s) \mathrm{d} s\right)^{2} \leq\left(\int_{a}^{b} f^{2}(s) \mathrm{d} s\right)\left(\int_{a}^{b} g^{2}(s) \mathrm{d} s\right) .
$$

Based on the above three lemmas, the following delayrange-dependent and rate-dependent stabilization criterion for the neutral-type neural network (6) can be investigated by constructing an appropriate Lyapunov-Krasovskii functional and applying the CCL technique. 
Theorem 4. For given scalars $h_{1}, h_{2}, h_{3}, \tau_{1}, \tau_{2}$, and $\tau_{3}$ such that $h_{2}>h_{1}>0$ and $\tau_{2}>\tau_{1}>0$, the system (6) subject to (3) is asymptotically stabilizable via the controller (7) if there exist real symmetry positive-definite matrices $P, \widetilde{P}, T_{i}, \widetilde{T}_{i}, Q_{i}(i=1, \ldots, 6), \widetilde{Q}_{4}, \widetilde{Q}_{5}, \widetilde{Q}_{6}, R_{j}(j=1, \ldots, 5)$, $\widetilde{R}_{4}, \widetilde{R}_{5}, M:=\operatorname{diag}\left(m_{1}, \ldots, m_{n}\right), N:=\operatorname{diag}\left(n_{1}, \ldots, n_{n}\right), \tilde{N}:=$ $\operatorname{diag}\left(\tilde{n}_{1}, \ldots, \tilde{n}_{n}\right)$, a matrix $\widetilde{K}$, and positive scalars $\varepsilon_{1}$ and $\varepsilon_{2}$, such that

$$
\begin{aligned}
& {\left[\begin{array}{ccccccc}
\tilde{\Pi} & \tilde{A}^{T} & \tilde{A}^{T} & \tilde{A}^{T} & \tilde{A}^{T} & \tilde{A}^{T} & \tilde{A}^{T} \\
\tilde{A} & -T_{1} & 0 & 0 & 0 & 0 & 0 \\
\tilde{A} & 0 & -T_{2} & 0 & 0 & 0 & 0 \\
\tilde{A} & 0 & 0 & -T_{3} & 0 & 0 & 0 \\
\tilde{A} & 0 & 0 & 0 & -T_{4} & 0 & 0 \\
\tilde{A} & 0 & 0 & 0 & 0 & -T_{5} & 0 \\
\tilde{A} & 0 & 0 & 0 & 0 & 0 & -T_{6}
\end{array}\right]<0,} \\
& {\left[\begin{array}{cc}
-\widetilde{T}_{1} & \widetilde{P} \\
\widetilde{P} & -\widetilde{Q}_{4}
\end{array}\right] \leq 0, \quad\left[\begin{array}{cc}
-\widetilde{T}_{2} & \widetilde{P} \\
\widetilde{P} & -\tau_{12}^{-1} \widetilde{R}_{4}
\end{array}\right] \leq 0,} \\
& {\left[\begin{array}{cc}
-\widetilde{T}_{3} & \widetilde{P} \\
\widetilde{P} & -\tau_{2}^{-1} \widetilde{R}_{5}
\end{array}\right] \leq 0} \\
& {\left[\begin{array}{cc}
-\widetilde{T}_{4} & \widetilde{P} \\
\widetilde{P} & -h_{12}^{-1} \widetilde{Q}_{5}
\end{array}\right] \leq 0, \quad\left[\begin{array}{cc}
-\widetilde{T}_{5} & \widetilde{P} \\
\widetilde{P} & -h_{2}^{-1} \widetilde{Q}_{6}
\end{array}\right] \leq 0,} \\
& {\left[\begin{array}{cc}
-\widetilde{T}_{6} & \widetilde{P} \\
\widetilde{P} & -\tilde{N}
\end{array}\right] \leq 0} \\
& P \widetilde{P}=I, \quad T_{i} \tilde{T}_{i}=I \quad(i=1, \ldots, 6), \\
& R_{4} \widetilde{R}_{4}=I, \quad R_{5} \widetilde{R}_{5}=I, \\
& Q_{4} \widetilde{Q}_{4}=I, \quad Q_{5} \widetilde{Q}_{5}=I, \\
& Q_{6} \widetilde{Q}_{6}=I, \quad N \tilde{N}=I,
\end{aligned}
$$

where

$$
\begin{aligned}
\tilde{\Pi}:= & e_{1}^{T}\left(\sum_{i=1}^{3} R_{i}+\sum_{i=1}^{3} Q_{i}+\varepsilon_{1} L^{T} L\right) e_{1} \\
& -e_{2}^{T}\left(\left(1-\tau_{3}\right) R_{3}-\varepsilon_{2} L^{T} L\right) e_{2}+\widetilde{A}^{T} e_{1}+e_{1}^{T} \tilde{A} \\
& -\left(1-h_{3}\right) e_{5}^{T} Q_{3} e_{5}-h_{12}^{-1}\left(e_{5}-e_{6}\right)^{T} Q_{6}\left(e_{5}-e_{6}\right) \\
& -\tau_{12}^{-1}\left(e_{2}-e_{4}\right)^{T} R_{5}\left(e_{2}-e_{4}\right) \\
& +e_{9}^{T}\left(M-\varepsilon_{1} I\right) e_{9}-e_{11}^{T} M e_{11}-e_{12}^{T} N e_{12} \\
& -\varepsilon_{2} e_{10}^{T} e_{10}-\left(1-h_{3}\right) e_{8}^{T} Q_{4} e_{8} \\
& -\tau_{12}^{-1}\left(e_{2}-e_{4}\right)^{T} R_{4}\left(e_{2}-e_{4}\right)
\end{aligned}
$$

$$
\begin{aligned}
& -\tau_{12}^{-1}\left(e_{2}-e_{3}\right)^{T} R_{4}\left(e_{2}-e_{3}\right) \\
& -e_{3}^{T} R_{1} e_{3}-e_{4}^{T} R_{2} e_{4} \\
& -\tau_{12}^{-1}\left(e_{2}-e_{3}\right)^{T} R_{5}\left(e_{2}-e_{3}\right) \\
& -\tau_{1}^{-1}\left(e_{1}-e_{3}\right)^{T} R_{5}\left(e_{1}-e_{3}\right) \\
& -e_{6}^{T} Q_{1} e_{6}-e_{7}^{T} Q_{2} e_{7} \\
& -h_{12}^{-1}\left(e_{5}-e_{6}\right)^{T} Q_{5}\left(e_{5}-e_{6}\right) \\
& -h_{12}^{-1}\left(e_{5}-e_{7}\right)^{T} Q_{6}\left(e_{5}-e_{7}\right) \\
& -h_{12}^{-1}\left(e_{5}-e_{7}\right)^{T} Q_{5}\left(e_{5}-e_{7}\right) \\
& -h_{1}^{-1}\left(e_{1}-e_{6}\right)^{T} Q_{6}\left(e_{1}-e_{6}\right) \text {, } \\
& \tilde{A}=[-P A+\tilde{K} 00000000 P D P B P C P E P F] \text {, } \\
& L=\operatorname{diag}\left(L_{1}, \ldots, L_{n}\right), \\
& e_{i}=\left[\begin{array}{lll}
0_{n \times(i-1)} & I_{n} & 0_{n \times(12-i)}
\end{array}\right] \quad(i=1, \ldots, 12), \\
& h_{12}=h_{2}-h_{1}, \quad \tau_{12}=\tau_{2}-\tau_{1} \text {. }
\end{aligned}
$$

Furthermore, when the LMIs (10)-(12) with the constraint (13) are feasible, a desired state feedback gain is given by $K=P^{-1} \tilde{K}$.

Proof. Choose a Lyapunov-Krasovskii functional as follows:

$$
V\left(y_{t}\right)=\sum_{i=1}^{6} V_{i}\left(y_{t}\right),
$$

with

$$
\begin{gathered}
V_{1}\left(y_{t}\right)=y^{T}(t) P y(t), \\
V_{2}\left(y_{t}\right)=\int_{t-h(t)}^{t}\left[y^{T}(s) Q_{3} y(s)+\dot{y}^{T}(s) Q_{4} \dot{y}(s)\right] \mathrm{d} s \\
+\sum_{i=1}^{2} \int_{t-h_{i}}^{t} y^{T}(s) Q_{i} y(s) \mathrm{d} s \\
+\int_{t-\tau(t)}^{t} y^{T}(s) R_{3} y(s) \mathrm{d} s+\sum_{i=1}^{2} \int_{t-\tau_{i}}^{t} y^{T}(s) R_{i} y(s) \mathrm{d} s, \\
V_{3}\left(y_{t}\right)=\int_{-\tau_{2}}^{-\tau_{1}} \int_{t+\theta}^{t} \dot{y}^{T}(s) R_{4} \dot{y}(s) \mathrm{d} s \mathrm{~d} \theta \\
+\int_{-\tau_{2}}^{0} \int_{t+\theta}^{t} \dot{y}^{T}(s) R_{5} \dot{y}(s) \mathrm{d} s \mathrm{~d} \theta, \\
V_{4}\left(y_{t}\right)=\int_{-h_{2}}^{-h_{1}} \int_{t+\theta}^{t} \dot{y}^{T}(s) Q_{5} \dot{y}(s) \mathrm{d} s \mathrm{~d} \theta \\
+\int_{-h_{2}}^{0} \int_{t+\theta}^{t} \dot{y}^{T}(s) Q_{6} \dot{y}(s) \mathrm{d} s \mathrm{~d} \theta,
\end{gathered}
$$




$$
\begin{gathered}
V_{5}\left(y_{t}\right)=\sum_{j=1}^{n} m_{j} \int_{0}^{\infty} z_{j}(\delta) \int_{t-\delta}^{t} g_{j}^{2}\left(y_{j}(\xi)\right) \mathrm{d} \xi \mathrm{d} \delta \\
V_{6}\left(y_{t}\right)=\sum_{j=1}^{n} n_{j} \int_{0}^{\infty} z_{j}(\delta) \int_{t-\delta}^{t} \dot{y}_{j}^{2}(\xi) \mathrm{d} \xi \mathrm{d} \delta .
\end{gathered}
$$

Set $\left\|y_{t}\right\|_{w}^{2}=\|y(t)\|^{2}+\int_{-\infty}^{0}\left\|y_{t}(\theta)\right\|^{2} d \theta+\int_{-\infty}^{0}\left\|\dot{y}_{t}(\theta)\right\|^{2} \mathrm{~d} \theta$. By some derivation, we have

$$
\lambda_{\min }(P)\|y(t)\|^{2} \leq V\left(y_{t}\right) \leq\left(a_{1}+a_{2}+a_{3}\right)\left\|y_{t}\right\|_{w}^{2},
$$

where

$$
\begin{aligned}
& a_{1}=\lambda_{\max }(P)+\lambda_{\max }\left(Q_{1}\right)+\lambda_{\max }\left(Q_{2}\right)+\lambda_{\max }\left(Q_{3}\right)+\lambda_{\max }\left(Q_{4}\right), \\
& a_{2}=\lambda_{\max }\left(R_{1}\right)+\lambda_{\max }\left(R_{2}\right)+\lambda_{\max }\left(R_{3}\right), \\
& a_{3}=\tau_{12} \lambda_{\max }\left(R_{4}\right)+\tau_{2} \lambda_{\max }\left(R_{5}\right)+h_{12} \lambda_{\max }\left(Q_{5}\right) \\
&+h_{2} \lambda_{\max }\left(Q_{6}\right)+\lambda_{\max }\left(M L^{T} L\right)+\lambda_{\max }(N),
\end{aligned}
$$

which implies that the functional $V\left(y_{t}\right)$ is a legitimate due to [26]. Next we deal with the derivatives of $V_{i}\left(y_{t}\right)(i=$ $1, \ldots, 6)$.

Set

$$
\begin{aligned}
& \widetilde{A}_{1}=\left[\begin{array}{llllllllllll}
-A+K & 0 & 0 & 0 & 0 & 0 & 0 & D & B & C & E & F
\end{array}\right], \\
& \xi(t)=\left[y^{T}(t), y^{T}(t-\tau(t)), y^{T}\left(t-\tau_{1}\right),\right. \\
& y^{T}\left(t-\tau_{2}\right), y^{T}(t-h(t)), \\
& y^{T}\left(t-h_{1}\right), y^{T}\left(t-h_{2}\right), \dot{y}^{T}(t-h(t)), \\
& g^{T}(y(t)), g^{T}(y(t-\tau(t))), \\
& \left(\int_{-\infty}^{t} Z(t-s) g(y(s)) \mathrm{d} s\right)^{T}, \\
& \left.\left(\int_{-\infty}^{t} Z(t-s) \dot{y}(s) \mathrm{d} s\right)^{T}\right]^{T} .
\end{aligned}
$$

When the controller (7) is applied to the system (6), the resultant closed-loop system is obtained as follows:

$$
\dot{y}(t)=\widetilde{A}_{1} \xi(t) .
$$

Due to Lemma 1, (3), the derivatives of $V_{i}\left(y_{t}\right)(i=1, \ldots, 4)$ along the trajectory of (21) are

$$
\begin{aligned}
\dot{V}_{1}\left(y_{t}\right) & =2 y^{T}(t) P \dot{y}(t)=2 y^{T}(t) P \widetilde{A}_{1} \xi(t) \\
& =\xi^{T}(t)\left(e_{1}^{T} P \widetilde{A}_{1}+\widetilde{A}_{1}^{T} P e_{1}\right) \xi(t),
\end{aligned}
$$

$$
\begin{aligned}
\dot{V}_{2}\left(y_{t}\right)= & y^{T}(t)\left(\sum_{i=1}^{3} Q_{i}+\sum_{i=1}^{3} R_{i}\right) y(t) \\
& -(1-\dot{h}(t)) y^{T}(t-h(t)) Q_{3} y(t-h(t)) \\
& -(1-\dot{h}(t)) \dot{y}^{T}(t-h(t)) Q_{4} \dot{y}(t-h(t)) \\
& -y^{T}\left(t-h_{1}\right) Q_{1} y\left(t-h_{1}\right) \\
& -y^{T}\left(t-h_{2}\right) Q_{2} y\left(t-h_{2}\right) \\
& -(1-\dot{\tau}(t)) y^{T}(t-\tau(t)) R_{3} y(t-\tau(t)) \\
& -y^{T}\left(t-\tau_{1}\right) R_{1} y\left(t-\tau_{1}\right) \\
& -y^{T}\left(t-\tau_{2}\right) R_{2} y\left(t-\tau_{2}\right)+\dot{y}^{T}(t) Q_{4} \dot{y}(t) \\
\leq & \xi^{T}(t) e_{1}^{T}\left(\sum_{i=1}^{3} Q_{i}+\sum_{i=1}^{3} R_{i}\right) e_{1} \xi(t)+\xi^{T}(t) \tilde{A}_{1}^{T} Q_{4} \tilde{A}_{1} \xi(t) \\
& -\xi^{T}(t) e_{7}^{T} Q_{2} e_{7} \xi(t) \\
& -\left(1-h_{3}\right) \xi^{T}(t) e_{8}^{T} Q_{4} e_{8} \xi(t)-\xi^{T}(t) e_{6}^{T} Q_{1} e_{6} \xi(t) \\
& -\left(1-h_{3}\right) \xi^{T}(t) e_{5}^{T} Q_{3} e_{5} \xi(t) \\
& -\left(1-\tau_{3}\right) \xi^{T}(t) e_{2}^{T} R_{3} e_{2} \xi(t)-\xi^{T}(t) e_{3}^{T} R_{1} e_{3} \xi(t) \\
& -\xi^{T}(t) e_{4}^{T} R_{2} e_{4} \xi(t),
\end{aligned}
$$

$$
\begin{aligned}
\dot{V}_{3}\left(y_{t}\right)= & \dot{y}^{T}(t)\left(\tau_{12} R_{4}+\tau_{2} R_{5}\right) \dot{y}(t) \\
& -\int_{t-\tau_{2}}^{t-\tau_{1}} \dot{y}^{T}(s) R_{4} \dot{y}(s) \mathrm{d} s-\int_{t-\tau_{2}}^{t} \dot{y}^{T}(s) R_{5} \dot{y}(s) \mathrm{d} s \\
= & \dot{y}^{T}(t)\left(\tau_{12} R_{4}+\tau_{2} R_{5}\right) \dot{y}(t)-\int_{t-\tau_{2}}^{t-\tau(t)} \dot{y}^{T}(s) R_{4} \dot{y}(s) \mathrm{d} s \\
& -\int_{t-\tau(t)}^{t-\tau_{1}} \dot{y}^{T}(s) R_{4} \dot{y}(s) \mathrm{d} s-\int_{t-\tau_{2}}^{t-\tau(t)} \dot{y}^{T}(s) R_{5} \dot{y}(s) \mathrm{d} s \\
& -\int_{t-\tau(t)}^{t-\tau_{1}} \dot{y}^{T}(s) R_{5} \dot{y}(s) \mathrm{d} s-\int_{t-\tau_{1}}^{t} \dot{y}^{T}(s) R_{5} \dot{y}(s) \mathrm{d} s \\
\leq & \xi^{T}(t) \tilde{A}_{1}^{T}\left(\tau_{12} R_{4}+\tau_{2} R_{5}\right) \tilde{A}_{1} \xi(t) \\
& -\tau_{12}^{-1} \xi^{T}(t)\left(e_{2}-e_{4}\right)^{T} R_{4}\left(e_{2}-e_{4}\right) \xi(t) \\
& -\tau_{12}^{-1} \xi^{T}(t)\left(e_{2}-e_{3}\right)^{T} R_{4}\left(e_{2}-e_{3}\right) \xi(t) \\
& -\tau_{12}^{-1} \xi^{T}(t)\left(e_{2}-e_{4}\right)^{T} R_{5}\left(e_{2}-e_{4}\right) \xi(t) \\
& -\tau_{12}^{-1} \xi^{T}(t)\left(e_{2}-e_{3}\right)^{T} R_{5}\left(e_{2}-e_{3}\right) \xi(t) \\
& -\tau_{1}^{-1} \xi^{T}(t)\left(e_{1}-e_{3}\right)^{T} R_{5}\left(e_{1}-e_{3}\right) \xi(t),
\end{aligned}
$$$$
\dot{V}_{4}\left(y_{t}\right)=\dot{y}^{T}(t)\left(h_{12} Q_{5}+h_{2} Q_{6}\right) \dot{y}(t)
$$$$
-\int_{t-h_{2}}^{t-h_{1}} \dot{y}^{T}(s) Q_{5} \dot{y}(s) \mathrm{d} s-\int_{t-h_{2}}^{t} \dot{y}^{T}(s) Q_{6} \dot{y}(s) \mathrm{d} s
$$ 


$$
\begin{aligned}
= & \dot{y}^{T}(t)\left(h_{12} Q_{5}+h_{2} Q_{6}\right) \dot{y}(t)-\int_{t-h_{2}}^{t-h(t)} \dot{y}^{T}(s) Q_{5} \dot{y}(s) \mathrm{d} s \\
& -\int_{t-h(t)}^{t-h_{1}} \dot{y}^{T}(s) Q_{5} \dot{y}(s) \mathrm{d} s-\int_{t-h_{2}}^{t-h(t)} \dot{y}^{T}(s) Q_{6} \dot{y}(s) \mathrm{d} s \\
& -\int_{t-h(t)}^{t-h_{1}} \dot{y}^{T}(s) Q_{6} \dot{y}(s) \mathrm{d} s-\int_{t-h_{1}}^{t} \dot{y}^{T}(s) Q_{6} \dot{y}(s) \mathrm{d} s \\
\leq & \xi^{T}(t) \tilde{A}_{1}^{T}\left(h_{12} Q_{5}+h_{2} Q_{6}\right) \tilde{A}_{1} \xi(t) \\
& -h_{12}^{-1} \xi^{T}(t)\left(e_{5}-e_{7}\right)^{T} Q_{5}\left(e_{5}-e_{7}\right) \xi(t) \\
& -h_{12}^{-1} \xi^{T}(t)\left(e_{5}-e_{6}\right)^{T} Q_{5}\left(e_{5}-e_{6}\right) \xi(t) \\
& -h_{12}^{-1} \xi^{T}(t)\left(e_{5}-e_{7}\right)^{T} Q_{6}\left(e_{5}-e_{7}\right) \xi(t) \\
& -h_{12}^{-1} \xi^{T}(t)\left(e_{5}-e_{6}\right)^{T} Q_{6}\left(e_{5}-e_{6}\right) \xi(t) \\
& -h_{1}^{-1} \xi^{T}(t)\left(e_{1}-e_{6}\right)^{T} Q_{6}\left(e_{1}-e_{6}\right) \xi(t) .
\end{aligned}
$$

By Lemma 3 and (4), we can obtain the derivatives of $V_{5}\left(y_{t}\right)$ and $V_{6}\left(y_{t}\right)$ as follows:

$$
\begin{aligned}
\dot{V}_{5}\left(y_{t}\right)= & \sum_{j=1}^{n} m_{j} \int_{0}^{\infty} z_{j}(\delta) g_{j}^{2}\left(y_{j}(t)\right) \mathrm{d} \delta \\
& -\sum_{j=1}^{n} m_{j} \int_{0}^{\infty} z_{j}(\delta) g_{j}^{2}\left(y_{j}(t-\delta)\right) \mathrm{d} \delta \\
= & \sum_{j=1}^{n} m_{j} g_{j}^{2}\left(y_{j}(t)\right) \int_{0}^{\infty} z_{j}(\delta) \mathrm{d} \delta-\sum_{j=1}^{n} m_{j} \int_{0}^{\infty} z_{j}(\delta) \mathrm{d} \delta \\
& \times \int_{0}^{\infty} z_{j}(\delta) g_{j}^{2}\left(y_{j}(t-\delta)\right) \mathrm{d} \delta \\
\leq & g^{T}(y(t)) M g(y(t)) \\
& -\sum_{j=1}^{n} m_{j}\left(\int_{0}^{\infty} z_{j}(\delta) g_{j}\left(y_{j}(t-\delta)\right) \mathrm{d} \delta\right)^{2} \\
= & g^{T}(y(t)) M g(y(t))-\left(\int_{-\infty}^{0} Z(t-s) g(y(s)) \mathrm{d} s\right)^{T} \\
& \times M\left(\int_{-\infty}^{0} Z(t-s) g(y(s)) \mathrm{d} s\right) \\
= & \xi^{T}(t) e_{9}^{T} M e_{9} \xi(t)-\xi^{T}(t) e_{11}^{T} M e_{11} \xi(t),
\end{aligned}
$$$$
\dot{V}_{6}\left(y_{t}\right) \leq \dot{y}^{T}(t) N \dot{y}(t)-\sum_{j=1}^{n} n_{j}\left(\int_{0}^{\infty} z_{j}(\delta) \dot{y}_{j}(t-\delta) \mathrm{d} \delta\right)^{2}
$$$$
=\xi^{T}(t) \widetilde{A}_{1}^{T} N \widetilde{A}_{1} \xi(t)-\xi^{T}(t) e_{12}^{T} N e_{12} \xi(t) .
$$

For the functions $g_{j}, j=1,2, \ldots, n$, using $g_{j}^{2}\left(\zeta_{j}\right) \leq L_{j}^{2} \zeta_{j}^{2}$, we get that

$$
\begin{aligned}
& 0 \leq-\varepsilon_{1} \xi^{T}(t) e_{9}^{T} e_{9} \xi(t)+\varepsilon_{1} \xi^{T}(t) e_{1}^{T} L^{T} L e_{1} \xi(t), \\
& 0 \leq-\varepsilon_{2} \xi^{T}(t) e_{10}^{T} e_{10} \xi(t)+\varepsilon_{2} \xi^{T}(t) e_{2}^{T} L^{T} L e_{2} \xi(t),
\end{aligned}
$$

where $L=\operatorname{diag}\left(L_{1}, \ldots, L_{n}\right)$. Noting that $K=P^{-1} \tilde{K}$, we have $\widetilde{A}=P \widetilde{A}_{1}$. The combination of (22)-(28) gives

$$
\dot{V}\left(y_{t}\right)=\sum_{i=1}^{6} \dot{V}_{i}\left(y_{t}\right) \leq \xi^{T}(t) \bar{\Pi} \xi(t),
$$

where

$$
\bar{\Pi}=\widetilde{\Pi}+\widetilde{A}_{1}^{T}\left(Q_{4}+\tau_{12} R_{4}+\tau_{2} R_{5}+h_{12} Q_{5}+h_{2} Q_{6}+N\right) \widetilde{A}_{1} .
$$

By Lemma 2 and (10)-(13), we can get that

$$
\left[\begin{array}{ccccccc}
\tilde{\Pi} & \tilde{A}_{1}^{T} P & \tilde{A}_{1}^{T} P & \tilde{A}_{1}^{T} P & \tilde{A}_{1}^{T} P & \tilde{A}_{1}^{T} P & \tilde{A}_{1}^{T} P \\
P \widetilde{A}_{1} & \Pi_{22} & 0 & 0 & 0 & 0 & 0 \\
P \widetilde{A}_{1} & 0 & \Pi_{33} & 0 & 0 & 0 & 0 \\
P \widetilde{A}_{1} & 0 & 0 & \Pi_{44} & 0 & 0 & 0 \\
P \widetilde{A}_{1} & 0 & 0 & 0 & \Pi_{55} & 0 & 0 \\
P \widetilde{A}_{1} & 0 & 0 & 0 & 0 & \Pi_{66} & 0 \\
P \widetilde{A}_{1} & 0 & 0 & 0 & 0 & 0 & \Pi_{77}
\end{array}\right]<0,
$$

where

$$
\begin{array}{ll}
\Pi_{22}=-P Q_{4}^{-1} P, & \Pi_{33}=-\tau_{12}^{-1} P R_{4}^{-1} P, \\
\Pi_{44}=-\tau_{2}^{-1} P R_{5}^{-1} P, & \Pi_{55}=-h_{12}^{-1} P Q_{5}^{-1} P, \\
\Pi_{66}=-h_{2}^{-1} P Q_{6}^{-1} P, & \Pi_{77}=-P N^{-1} P .
\end{array}
$$

Thus it is easy to see that $\dot{V}\left(y_{t}\right)<0$, which, together with (7) and [26, Theorem 3.1.6], implies that the system (6) is asymptotically stabilizable. The proof is completed.

Remark 5. A delay-range-dependent and rate-dependent condition under which the system (6) is asymptotically stabilizable is investigated in Theorem 4 . In $[7,8]$, the stability of a class of the neural networks with unbounded distributed delays has been studied; however, the neutral term was not considered in their models. In [18], only a unbounded distributed delay is taken into account. But in this paper the neutral-type neural networks with two unbounded distributed delays are studied. Therefore, Theorem 4 in this paper can be viewed as an extension of the corresponding results in $[7,8,18]$.

Remark 6. In order to apply [26, Theorem 3.1.6] to conclude that the system (6) is asymptotically stabilizable, we denote a new norm $\|\cdot\|_{w}^{2}$ on a space of functions.

Noting that the stabilization criterion proposed in Theorem 4 includes the nonlinear constraint (13), we cannot obtain the desired gain $K$ by using the LMI Controller Toolbox of MATLAB. In order to deal with the nonlinear constraint (13), we offer the following CCL algorithm. 
The algorithm is available to determine the maximum value of $h_{2}$ for given $h_{1}, h_{3}, \tau_{1}, \tau_{2}$, and $\tau_{3}$ and the corresponding gain $K$.

Algorithm 7 (CCL algorithm). Step 1. Choose a sufficiently small initial $h_{2}>h_{1}$, such that there exists a feasible solution to (10)-(12) and

$$
\begin{aligned}
& {\left[\begin{array}{cc}
P & I \\
I & \widetilde{P}
\end{array}\right] \geq 0, \quad\left[\begin{array}{cc}
N & I \\
I & \tilde{N}
\end{array}\right] \geq 0, \quad\left[\begin{array}{cc}
R_{4} & I \\
I & \widetilde{R}_{4}
\end{array}\right] \geq 0,} \\
& {\left[\begin{array}{cc}
R_{5} & I \\
I & \widetilde{R}_{5}
\end{array}\right] \geq 0, \quad\left[\begin{array}{cc}
T_{i} & I \\
I & \widetilde{T}_{i}
\end{array}\right] \geq 0 \quad(i=1, \ldots, 6),} \\
& {\left[\begin{array}{cc}
Q_{j} & I \\
I & \widetilde{Q}_{j}
\end{array}\right] \geq 0 \quad(j=4,5,6) .}
\end{aligned}
$$

Set $h_{\max }=h_{2}$.

Step 2. Find a feasible set of $P_{0}, \widetilde{P}_{0}, N_{0}, \tilde{N}_{0}, T_{i 0}, \widetilde{T}_{i 0}(i=$ $1, \ldots, 6), R_{l 0}, \widetilde{R}_{l 0}(l=4,5), Q_{j 0}$, and $\widetilde{Q}_{j 0}(j=4,5,6)$ satisfying (10)-(12), (33). Set $k=0$.

Step 3. Solve the following LMI problem for the variables $\widetilde{K}, P, \widetilde{P}, N, \tilde{N}, T_{i}, \widetilde{T}_{i}(i=1, \ldots, 6), R_{l}, \widetilde{R}_{l}(l=4,5), Q_{j}$, and $\widetilde{Q}_{j}(j=4,5,6)$ :

$$
\min _{\text {subject to }(8)-(10),(29)} \operatorname{tr} \Theta_{k}
$$

where

$$
\begin{aligned}
\Theta_{k}= & P_{k} \tilde{P}+P \widetilde{P}_{k}+N_{k} \tilde{N}+N \tilde{N}_{k}+\sum_{i=1}^{6}\left(T_{i} \widetilde{T}_{i k}+T_{i k} \widetilde{T}_{i}\right) \\
& +\sum_{l=4}^{5}\left(R_{l} \widetilde{R}_{l k}+R_{l k} \widetilde{R}_{l}\right)+\sum_{j=4}^{6}\left(Q_{j} \widetilde{Q}_{j k}+Q_{j k} \widetilde{Q}_{j}\right) .
\end{aligned}
$$

Set $P_{k+1}=P, \widetilde{P}_{k+1}=\widetilde{P}, N_{k+1}=N, \tilde{N}_{k+1}=\tilde{N}, T_{i k+1}=T_{i}$, $\widetilde{T}_{i k+1}=\widetilde{T}_{i}(i=1, \ldots, 6), R_{l k+1}=R_{l}, \widetilde{R}_{l k+1}=\widetilde{R}_{l}(l=4,5)$, $Q_{j k+1}=Q_{j}$ and $\widetilde{Q}_{j k+1}=\widetilde{Q}_{j}(j=4,5,6)$.

Step 4. If the LMI.

$$
\hat{\Pi}:=\left[\begin{array}{ccccccc}
\tilde{\Pi} & \tilde{A}_{1}^{T} Q_{4} & \tilde{A}_{1}^{T} R_{4} & \tilde{A}_{1}^{T} R_{5} & \tilde{A}_{1}^{T} Q_{5} & \tilde{A}_{1}^{T} Q_{6} & \tilde{A}_{1}^{T} N \\
Q_{4} \tilde{A}_{1} & -Q_{4} & 0 & 0 & 0 & 0 & 0 \\
R_{4} \tilde{A}_{1} & 0 & -\tau_{12}^{-1} R_{4} & 0 & 0 & 0 & 0 \\
R_{5} \tilde{A}_{1} & 0 & 0 & -\tau_{2}^{-1} R_{5} & 0 & 0 & 0 \\
Q_{5} \tilde{A}_{1} & 0 & 0 & 0 & -h_{12}^{-1} Q_{5} & 0 & 0 \\
Q_{6} \tilde{A}_{1} & 0 & 0 & 0 & 0 & -h_{2}^{-1} Q_{6} & 0 \\
N \tilde{A}_{1} & 0 & 0 & 0 & 0 & 0 & -N
\end{array}\right]<0
$$

is feasible for the variables $N, T_{i}(i=1, \ldots, 6), R_{l}(l=4,5)$, and $Q_{j}(j=4,5,6)$ and the matrices $\widetilde{K}$ and $P$ obtained in Step 3, then set $K=P^{-1} \tilde{K}$ and stop. If the LMI (36) is infeasible within a specified number of iterations, then stop; otherwise, set $k=k+1$ and go to Step 3 .

Remark 8. The idea of the above algorithm is taken from [27]. Compared with the CCL algorithms proposed in [28, 29], the merit of the above algorithm is that more freedoms are provided to test the iteration stop conditions (see [27, Remark 4] for details).

Next, we consider the following neutral-type neural network (37), which is a special case of (6). A stability criterion for the neural network (37) has been presented in [21]. The following corollary can be immediately obtained from Theorem 4, which gives a stabilization criterion for the neutral-type neural network (37):

$$
\begin{aligned}
\dot{y}(t)= & -A y(t)+B g(y(t)) \\
& +C g(y(t-h(t)))+D \dot{y}(t-h(t))
\end{aligned}
$$

$$
\begin{aligned}
& +E \int_{-\infty}^{t} Z(t-s) g(y(s)) \mathrm{d} s \\
& +F \int_{-\infty}^{t} Z(t-s) \dot{y}(s) \mathrm{d} s+I(t),
\end{aligned}
$$

where

$$
0 \leq h(t) \leq h_{2}, \quad \dot{h}(t) \leq h_{3} .
$$

Corollary 9. For given scalars $h_{2}$ and $h_{3}$ such that $h_{2}>0$, the neutral-type neural network (37) subject to (38) is asymptotically stabilizable via the controller (7) if there exist real symmetry positive-definite matrices $P, \widetilde{P}, Q_{i}(i=1, \ldots, 4)$, $\widetilde{Q}_{2}, \widetilde{Q}_{4}, T_{j}, \widetilde{T}_{j}(j=1,2,3), M:=\operatorname{diag}\left(m_{1}, \ldots, m_{n}\right) \mid, N:=$ $\operatorname{diag}\left(n_{1}, \ldots, n_{n}\right)$, and $\tilde{N}:=\operatorname{diag}\left(\tilde{n}_{1}, \ldots, \tilde{n}_{n}\right)$, a matrix $\tilde{K}$ and positive scalars $\varepsilon_{1}$ and $\varepsilon_{2}$, such that

$$
\Sigma:=\left[\begin{array}{cccc}
\tilde{\Sigma} & \mathcal{A}^{T} & \mathcal{A}^{T} & \mathcal{A}^{T} \\
\mathcal{A} & -T_{1} & 0 & 0 \\
\mathcal{A} & 0 & -T_{2} & 0 \\
\mathcal{A} & 0 & 0 & -T_{3}
\end{array}\right]<0,
$$




$$
\begin{gathered}
{\left[\begin{array}{cc}
-\widetilde{T}_{1} & \widetilde{P} \\
\widetilde{P} & -\widetilde{Q}_{2}
\end{array}\right] \leq 0, \quad\left[\begin{array}{cc}
-\widetilde{T}_{2} & \widetilde{P} \\
\widetilde{P} & -h_{2}^{-1} \widetilde{Q}_{4}
\end{array}\right] \leq 0,} \\
{\left[\begin{array}{cc}
-\widetilde{T}_{3} & \widetilde{P} \\
\widetilde{P} & -\widetilde{N}
\end{array}\right] \leq 0} \\
P \widetilde{P}=I, \quad T_{j} \widetilde{T}_{j}=I \quad(j=1,2,3), \\
Q_{2} \widetilde{Q}_{2}=I, \quad Q_{4} \widetilde{Q}_{4}=I, \quad N \tilde{N}=I
\end{gathered}
$$

where

$$
\begin{aligned}
& \tilde{\Sigma}:=\hat{e}_{1}^{T}\left(Q_{1}+Q_{3}+\varepsilon_{1} L^{T} L\right) \hat{e}_{1} \\
& -\hat{e}_{2}\left(\left(1-h_{3}\right) Q_{1}-\varepsilon_{2} L^{T} L\right) \hat{e}_{2}+\mathcal{A}^{T} \hat{e}_{1} \\
& +\hat{e}_{1}^{T} \mathcal{A}-\left(1-h_{3}\right) \hat{e}_{4}^{T} Q_{2} \hat{e}_{4} \\
& -\hat{e}_{3}^{T} Q_{3} \hat{e}_{3}+\hat{e}_{5}^{T}\left(M-\varepsilon_{1} I\right) \hat{e}_{5}-\hat{e}_{7}^{T} M \hat{e}_{7} \\
& -h_{2}^{-1}\left(\hat{e}_{2}-\hat{e}_{3}\right)^{T} Q_{4}\left(\hat{e}_{2}-\hat{e}_{3}\right) \\
& -h_{2}^{-1}\left(\hat{e}_{1}-\hat{e}_{2}\right)^{T} Q_{4}\left(\hat{e}_{1}-\hat{e}_{2}\right) \\
& -\hat{e}_{8}^{T} N \hat{e}_{8}-\varepsilon_{2} \hat{e}_{6}^{T} \hat{e}_{6} \text {, } \\
& \mathcal{A}=\left[\begin{array}{llllllll}
-P A+\widetilde{K} & 0 & 0 & P D & P B & P C & P E & P F
\end{array}\right] \text {, } \\
& \hat{e}_{i}=\left[\begin{array}{lll}
0_{n \times(i-1)} & I_{n} & 0_{n \times(8-i)}
\end{array}\right], \quad i=1, \ldots, 8 .
\end{aligned}
$$

Furthermore, when the LMIs (39) with the constraint (40) are feasible, a desired state feedback gain is given by $K=P^{-1} \widetilde{K}$.

Proof. If we use the following $\hat{\xi}(t)$ and $\hat{V}\left(y_{t}\right)$ instead of $\xi(t)$ and $V\left(y_{t}\right)$ in the proof of Theorem 4, respectively, then the proof can be easily completed:

$$
\hat{V}\left(y_{t}\right)=\sum_{i=1}^{5} \widehat{V}_{i}\left(y_{t}\right)
$$

with

$$
\begin{gathered}
\hat{V}_{1}\left(y_{t}\right)=y^{T}(t) P y(t), \\
\hat{V}_{2}\left(y_{t}\right)=\int_{t-h(t)}^{t}\left[y^{T}(s) Q_{1} y(s)+\dot{y}^{T}(s) Q_{2} \dot{y}(s)\right] \mathrm{d} s \\
+\int_{t-h_{2}}^{t} y^{T}(s) Q_{3} y(s) \mathrm{d} s, \\
\hat{V}_{3}\left(y_{t}\right)=\int_{-h_{2}}^{0} \int_{t+\theta}^{t} \dot{y}^{T}(s) Q_{4} \dot{y}(s) \mathrm{d} s \mathrm{~d} \theta, \\
\hat{V}_{4}\left(y_{t}\right)=\sum_{j=1}^{n} m_{j} \int_{0}^{\infty} Z_{j}(\delta) \int_{t-\delta}^{t} g_{j}^{2}\left(y_{j}(\xi)\right) \mathrm{d} \xi \mathrm{d} \delta, \\
\hat{V}_{5}\left(y_{t}\right)=\sum_{j=1}^{n} n_{j} \int_{0}^{\infty} Z_{j}(\delta) \int_{t-\delta}^{t} \dot{y}_{j}^{2}(\xi) \mathrm{d} \xi \mathrm{d} \delta .
\end{gathered}
$$

$$
\begin{aligned}
& \hat{\xi}(t)=\left[y^{T}(t), y^{T}(t-h(t)), y^{T}\left(t-h_{2}\right),\right. \\
& \dot{y}^{T}(t-h(t)), g^{T}(y(t)), g^{T}(y(t-h(t))), \\
&\left(\int_{-\infty}^{t} Z(t-s) g(y(s)) \mathrm{d} s\right)^{T}, \\
&\left.\left(\int_{-\infty}^{t} Z(t-s) \dot{y}(s) \mathrm{d} s\right)^{T}\right]^{T} .
\end{aligned}
$$

The following system model is a practical partial element equivalent circuit (PEEC) that is described in [30, Figure 1] by Bellen et al.:

$$
\begin{gathered}
\dot{y}(t)=A y(t)+B y(\mathrm{t}-\tau)+C \dot{y}(t-\tau)+I(t), \\
y(\theta)=\phi(\theta), \quad \forall \theta \in[-\tau, 0],
\end{gathered}
$$

where $\tau$ is a positive scalar representing the system delay.

It should be emphasized that the matrix $A$ in (44) is not necessarily diagonal, which is different from the one in (6). However, one can easily find that the proof of Theorem 4 is always available whether the matrix $A$ is diagonal or not. For this reason, we can derive the following Corollaries 10 and 12 by a method similar to obtaining Theorem 4 .

Corollary 10. For a given scalar $\tau>0$, the neutral-type system (44) is asymptotically stabilizable via the controller (7) if there exist real symmetry positive-definite matrices $P, \widetilde{P}, Q_{i}(i=$ $1, \ldots, 3), \widetilde{Q}_{2}, \widetilde{Q}_{3}, T_{j}$, and $\widetilde{T}_{j}(j=1,2)$ and a matrix $\widetilde{K}$, such that

$$
\begin{gathered}
\Xi:=\left[\begin{array}{ccc}
\widetilde{\Xi} & \AA^{T} & \check{A}^{T} \\
\check{A} & -T_{1} & 0 \\
\check{A} & 0 & -T_{2}
\end{array}\right]<0, \\
{\left[\begin{array}{cc}
-\widetilde{T}_{1} & \widetilde{P} \\
\widetilde{P} & -\widetilde{Q}_{2}
\end{array}\right] \leq} \\
0, \quad\left[\begin{array}{cc}
-\widetilde{T}_{2} & \widetilde{P} \\
\widetilde{P} & -\tau^{-1} \widetilde{Q}_{3}
\end{array}\right] \leq 0, \\
\\
P \widetilde{P}=I, \quad T_{j} \widetilde{T}_{j}=I \quad(j=1,2), \\
Q_{2} \widetilde{Q}_{2}=I, \quad Q_{3} \widetilde{Q}_{3}=I,
\end{gathered}
$$

where

$$
\begin{aligned}
\check{\Xi}:= & \check{e}_{1}^{T} Q_{1} \check{e}_{1}-\check{e}_{2}^{T} Q_{1} \check{e}_{2}+\check{A}^{T} \check{e}_{1}+\check{e}_{1}^{T} \check{A} \\
& -\check{e}_{3}^{T} Q_{2} \check{e}_{3}-\tau^{-1}\left(\check{e}_{1}-\check{e}_{2}\right)^{T} Q_{3}\left(\check{e}_{1}-\check{e}_{2}\right), \\
\check{A}= & {\left[\begin{array}{lll}
P A+\widetilde{K} & P B & P C
\end{array}\right], } \\
\check{e}_{i}= & {\left[\begin{array}{lll}
0_{n \times(i-1)} & I_{n} & 0_{n \times(3-i)}
\end{array}\right], \quad i=1,2,3 . }
\end{aligned}
$$

Furthermore, when the LMIs (46) and (47) with the nonlinear constraint (48) are feasible, a desired state feedback gain is given by $K=P^{-1} \widetilde{K}$. 


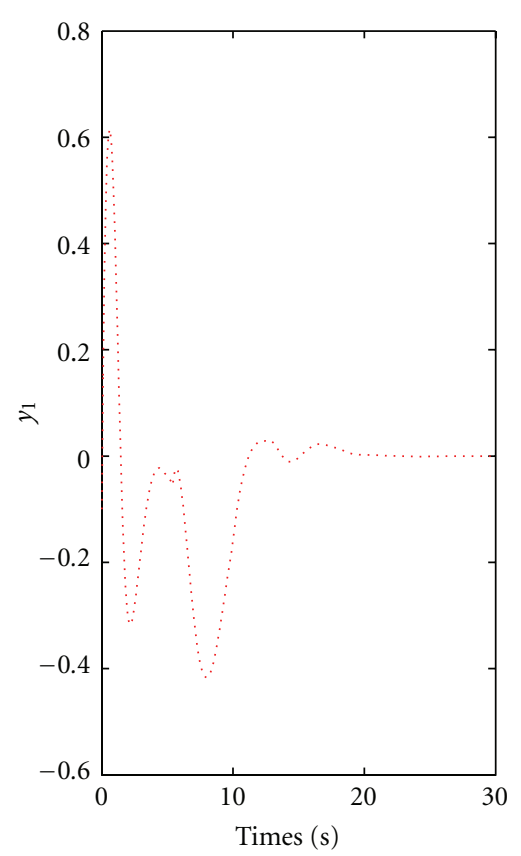

(a)

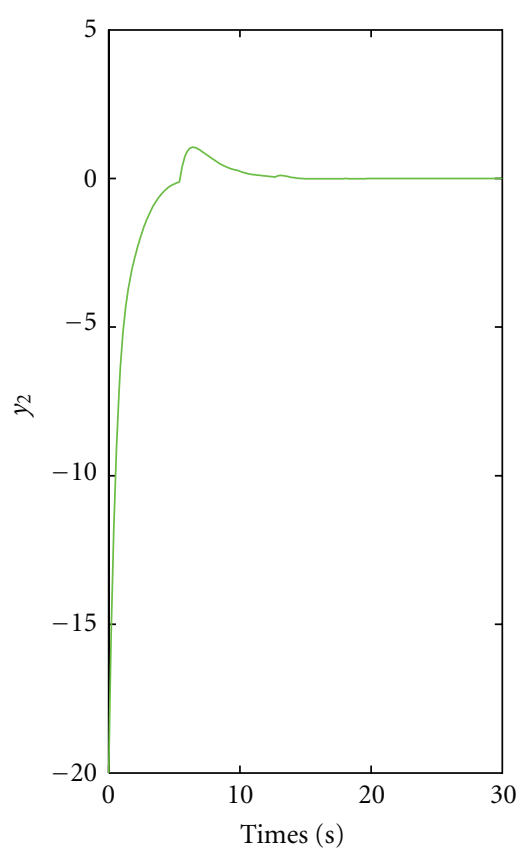

(b)

FIgURE 1: State responses of the closed-loop system for $z_{1}(s)=z_{2}(s)=1-|s-1|, 0 \leq s \leq 2$.

Remark 11. Similar to Algorithm 7, one can solve the stabilization criteria proposed in Corollaries 9 and 10.

Corollary 12. For a given scalar $\tau>0$, the unforced system of (44) is asymptotically stable if there exist real symmetry positive-definite matrices $P$ and $Q_{i}(i=1,2,3)$ such that

$$
\begin{aligned}
\Theta:= & \check{e}_{1}^{T} Q_{1} \check{e}_{1}-\check{e}_{2}^{T} Q_{1} \check{e}_{2}+\bar{A}^{T} P \check{e}_{1}+\check{e}_{1}^{T} P \bar{A}-\check{e}_{3}^{T} Q_{2} \check{e}_{3} \\
& -\tau^{-1}\left(\check{e}_{1}-\check{e}_{2}\right)^{T} Q_{3}\left(\check{e}_{1}-\check{e}_{2}\right)+\bar{A}^{T}\left(Q_{2}+\tau Q_{3}\right) \bar{A}<0,
\end{aligned}
$$

where

$$
\bar{A}=\left[\begin{array}{lll}
A & B & C
\end{array}\right],
$$

and $\check{e}_{i}$ is defined as in Corollary 10.

\section{Numerical Examples}

In this section, we will illustrate our approach by several numerical examples.

Example 13. Consider the neutral-type neural network (6), where

$$
\begin{gathered}
A=\left[\begin{array}{ll}
2 & 0 \\
0 & 3
\end{array}\right], \quad B=\left[\begin{array}{cc}
-0.34 & 0 \\
-0.1 & 0.1
\end{array}\right], \\
C=\left[\begin{array}{cc}
0.1 & 0.2 \\
-0.15 & -0.18
\end{array}\right], \quad D=\left[\begin{array}{cc}
0.1 & 0 \\
0 & 0.1
\end{array}\right], \\
E=\left[\begin{array}{cc}
0.41 & -0.5 \\
0.69 & 0.31
\end{array}\right], \quad F=\left[\begin{array}{cc}
0.3 & -0.15 \\
0.5 & -0.2
\end{array}\right],
\end{gathered}
$$

$$
\begin{gathered}
L_{j}=1, \quad j=1,2, \\
\tau_{3}=0.5, \quad \tau_{2}=4.8, \quad \tau_{1}=4.1 \\
h_{3}=0.5, \quad h_{1}=4.5, \quad h_{2}=7.5, g(y(s))=\tanh \left(\frac{y(s)}{2}\right) .
\end{gathered}
$$

Solving the LMIs (10)-(12) with nonlinear constraint (13) by Algorithm 7 and the LMI Control Toolbox of MATLAB, we can get a desired state feedback gain stabilizing the system as follows:

$$
K=\left[\begin{array}{cc}
0.7763 & -0.1070 \\
-0.1070 & 1.7242
\end{array}\right]
$$

The state response curves of the closed-loop system are given in Figures 1 and 2, where the initial functions in Figures 1 and 2 are, respectively, chosen as $\phi(\theta)=\left[\begin{array}{l}-0.1 \\ -20\end{array}\right]$, $\theta \in(-\infty, 0]$ and $\phi(\theta)=\left[\begin{array}{c}e^{\theta} \\ e^{2 \theta}\end{array}\right], \theta \in(-\infty, 0]$.

Example 14. Consider the system (37) with the following parameter matrices:

$$
\begin{array}{ll}
A=\left[\begin{array}{cc}
3.6 & 0 \\
0 & 3.6
\end{array}\right], & B=\left[\begin{array}{cc}
1.198 & 0.1 \\
0.1 & 1.198
\end{array}\right], \\
C=\left[\begin{array}{cc}
0.1 & 0.16 \\
0.05 & 0.1
\end{array}\right], & D=\left[\begin{array}{cc}
0.2 & 0 \\
0 & 0.2
\end{array}\right], \\
E=\left[\begin{array}{cc}
0.4 & -0.2 \\
0.3 & 0.2
\end{array}\right], & F=\left[\begin{array}{cc}
0.3 & -0.15 \\
0.5 & -0.2
\end{array}\right],
\end{array}
$$




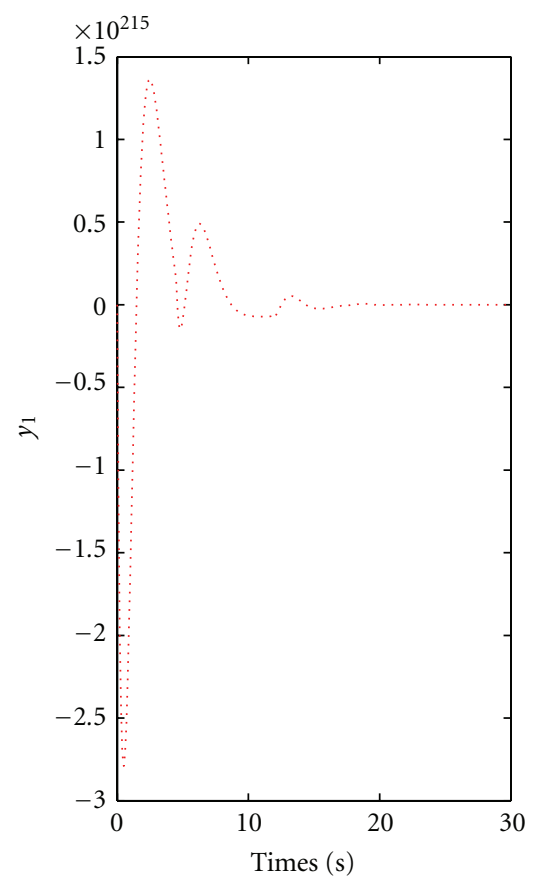

(a)

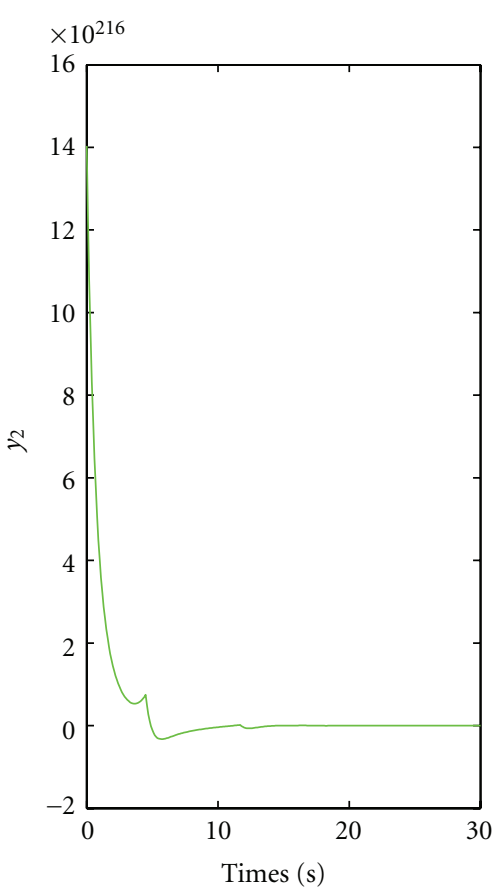

(b)

Figure 2: State responses of the closed-loop system for $z_{1}(s)=z_{2}(s)=(\sqrt{2} / \pi) e^{-(1 / 2) s^{2}}, s \in(-\infty, 0]$.

$$
\begin{aligned}
& L_{j}=1, \quad j=1,2, \quad \phi(\theta)=\left[\begin{array}{l}
-0.1 \\
-20
\end{array}\right], \\
& \theta \in(-\infty, 0], g(y(s))=\tanh \left(\frac{y(s)}{2}\right), \\
& z(s)=1-|s-1| \quad(0 \leq s \leq 2), \quad h_{2}=8 .
\end{aligned}
$$

When $h_{3}=0.15$ by the LMI Control Toolbox of MATLAB, it can be verified that the LMI in [21, Theorem 3.1] is not feasible, so it cannot guarantee that the unforced system is stable. Now we check the stabilization condition proposed in Corollary 9 of this paper and find that it is feasible. So, by Corollary 9 the system is asymptotically stabilizable by state feedback controller, and a desired state feedback gain is given by

$$
K=\left[\begin{array}{cc}
1.6655 & -0.3168 \\
-0.3168 & 1.8069
\end{array}\right]
$$

and, further, the state response curves of the closed-loop system are given in Figure 3.

When $h_{3}=0$, by the LMI Control Toolbox of MATLAB, it can be verified that the LMI in [21, Theorem 3.2] is not feasible, which cannot guarantee the unforced system is stable. However, the stabilization condition proposed in Corollary 9 can be satisfied. Hence, by Corollary 9, the neutral-type neural network is asymptotically stabilizable via state feedback controller, and a desired state feedback controller gain is given by

$$
K=\left[\begin{array}{cc}
1.6847 & -0.3110 \\
-0.3110 & 1.8198
\end{array}\right] \text {, }
$$

and, further, the state response curves of the closed-loop system are given in Figure 4.

Example 15. Consider the PEEC system (44) with the following parameter matrices:

$$
\begin{gathered}
A=100 \times\left[\begin{array}{ccc}
\beta & 1 & 2 \\
3 & -9 & 0 \\
1 & 2 & -6
\end{array}\right], \quad B=100 \times\left[\begin{array}{ccc}
1 & 0 & -3 \\
-0.5 & -0.5 & -1 \\
-0.5 & -1.5 & 0
\end{array}\right], \\
C=\frac{1}{72}\left[\begin{array}{ccc}
-1 & 5 & 2 \\
4 & 0 & 3 \\
-2 & 4 & 1
\end{array}\right] .
\end{gathered}
$$

Applying Corollaries 12 and 10, the maximum delay bounds $\tau_{M}$ for the various $\beta$ are presented in Table 1 , which clearly shows the effectiveness of our approach. Through Table 1, we can get that the stability criterion proposed in this paper is less conservative than the ones in [31-33].

The following example is provided for applying the approach proposed in this paper to a realistic problem which is motivated by the small PEEC model in [30, Figure 1]. 


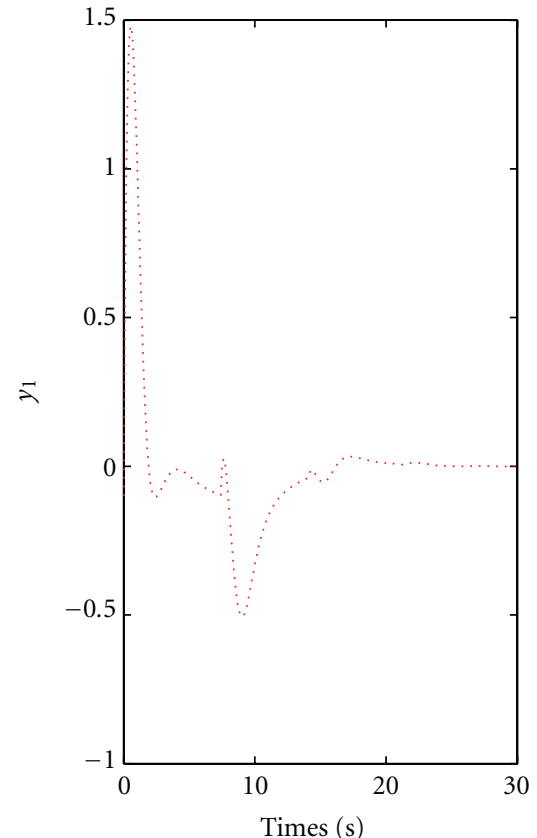

(a)

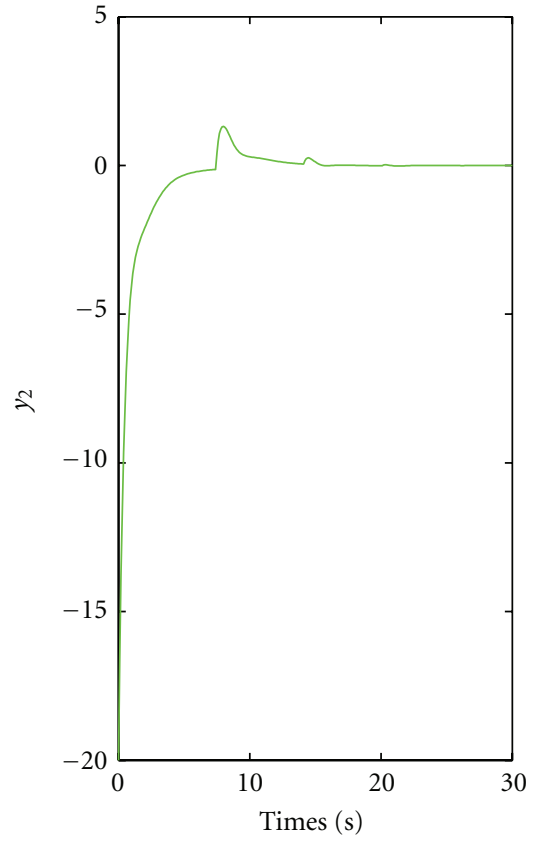

(b)

Figure 3: State responses of the closed-loop system when $h_{3}=0.15$.

TABLE 1: Allowable upper bound of $\tau_{M}$ for different $\beta$.

\begin{tabular}{|c|c|c|c|c|c|}
\hline$\beta$ & -2.121 & -2.122 & -2.123 & -2.124 & -2.125 \\
\hline [31, Theorem 3.1] & 220.2 & 220.1 & 201.0101 & 202.5203 & 163.2513 \\
\hline$[32$, Theorem 1] & 401.5 & 401.4764 & 401.4507 & 401.4251 & 401.3979 \\
\hline$[33$, Theorem 1] & 2202.57 & 2200.34 & 2198.331 & 2214.12 & 2229.1 \\
\hline Corollary 12 in this paper & 3000.1 & 3000.11 & 3000.1 & 3000.1 & 3000.11 \\
\hline Corollary 10 in this paper & 11000 & 9000 & 6000 & 6000 & 8000 \\
\hline
\end{tabular}

Example 16. Consider the PEEC system (44) with

$$
\begin{gathered}
A=100 \times\left[\begin{array}{ccc}
-7 & 1 & 2 \\
3 & -9 & 0 \\
1 & 2 & -6
\end{array}\right], \quad B=100 \times\left[\begin{array}{ccc}
1 & 0 & -3 \\
-0.5 & -0.5 & -1 \\
-0.5 & -1.5 & 0
\end{array}\right], \\
C=\frac{1}{72}\left[\begin{array}{ccc}
-1 & 5 & 2 \\
4 & 0 & 3 \\
-2 & 4 & 1
\end{array}\right], \quad \tau=1 .
\end{gathered}
$$

When $I(t) \equiv 0$, Bellen et al. [30] gave sufficient conditions for the asymptotic stability of the zero solution to (44) by utilizing a suitable reformulation of the system.

By applying Algorithm 7 and the LMI Control Toolbox of MATLAB to solve LMIs (46) and (47) with the nonlinear constraint (48), a required state feedback gain is obtained as follows:

$$
K=\left[\begin{array}{ccc}
66.1662 & -283.9848 & -0.7157 \\
-487.7185 & 45.6016 & -31.1715 \\
-74.6213 & -146.4500 & 1.7714
\end{array}\right]
$$

The simulation results for the PEEC system are shown by Figures 5 and 6 with the initial value $\phi(\theta)=$ $[\sin (t) \sin (2 t) \sin (3 t)]^{T}$. Figure 5 represents the state responses of the PEEC system when $I(t) \equiv 0$, and Figure 6 represents the state responses of the resultant closed-loop system. From Figures 5 and 6, we can see that both the openloop and closed-loop systems are stable, however, the speed of convergence towards the null point in the closed-loop system is faster than one in the open-loop system.

\section{Conclusions}

In this paper, the delay-range-dependent and rate-dependent stabilization criteria for a class of neutral-type neural networks with time-varying discrete and unbounded distributed delays are established. The criteria are derived by constructing an appropriate Lyapunov-Krasovskii functional and using certain matrix technique. A CCL algorithm is developed to obtain the state feedback gain $K$. Numerical examples are provided to show that our results are more suitable than some existing ones, which illustrate the merits of the proposed approach. 


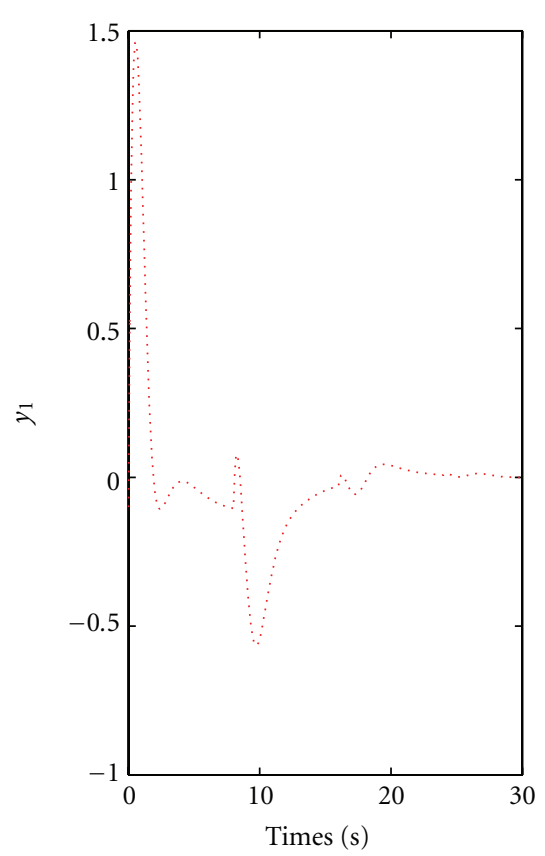

(a)

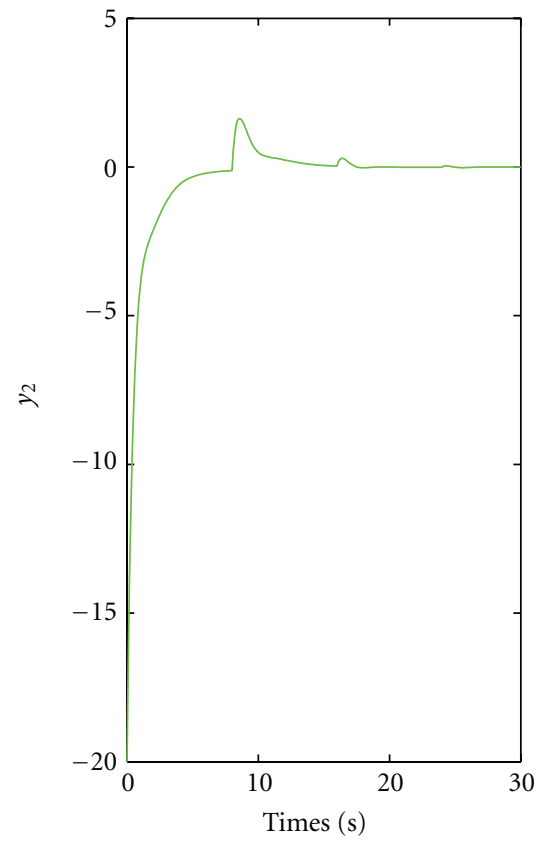

(b)

FIGURE 4: State responses of the closed-loop system when $h_{3}=0$.

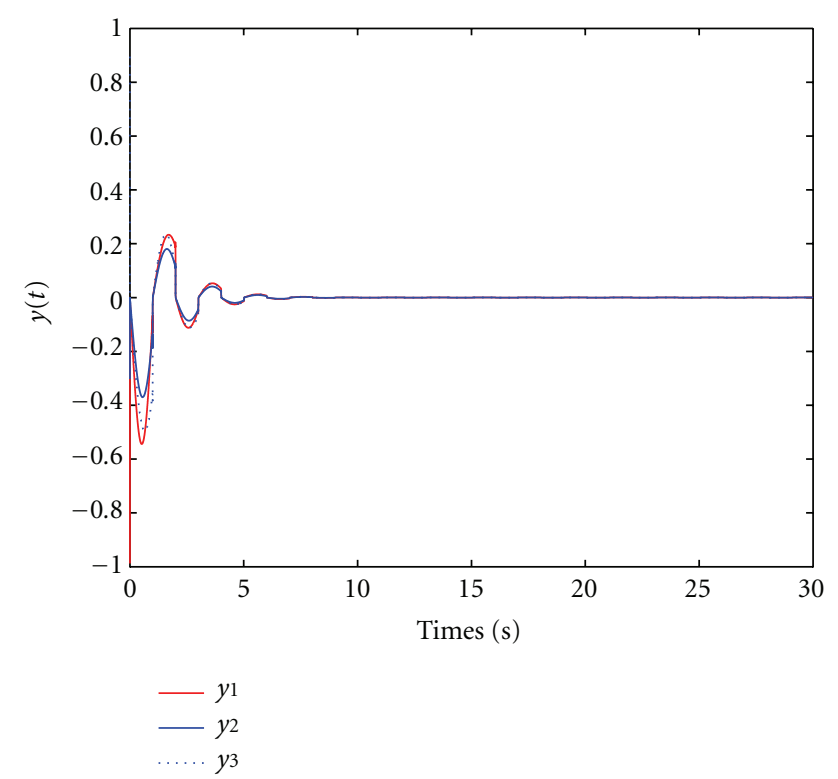

FIGURE 5: State responses of the open-loop system.

\section{Acknowledgments}

This work is supported by the fund of Heilongjiang Education Committee under Grant no. 12521429 and the fund of Heilongjiang University Innovation Team Support Plan under Grant no. Hdtd2010-03. The authors thank the anonymous referees for their helpful comments and suggestions that improved greatly this paper.

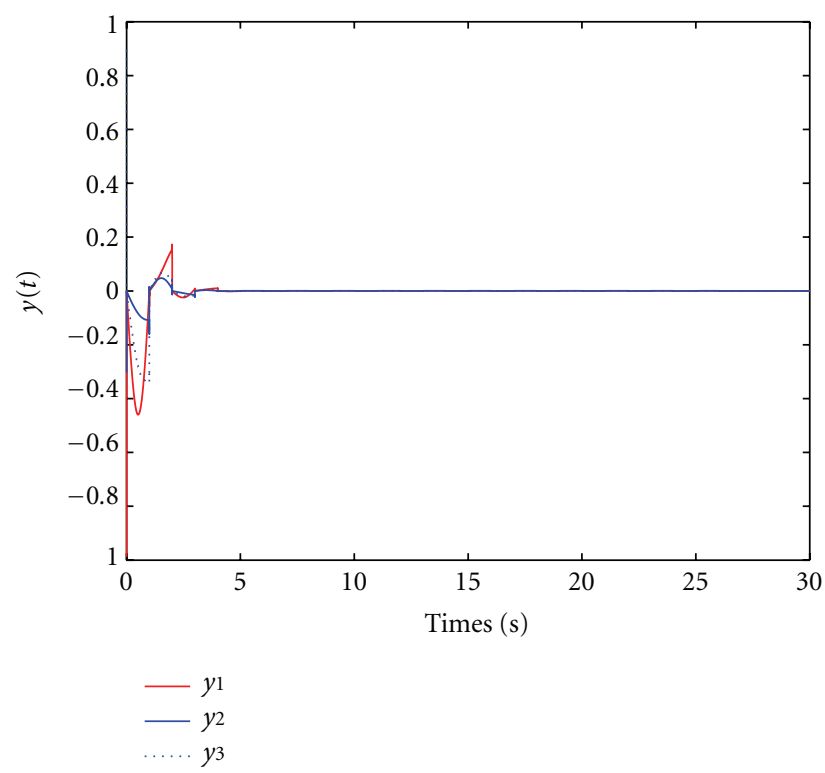

Figure 6: State responses of the closed-loop system.

\section{References}

[1] W. S. McCulloch and W. Pitts, "A logical calculus of the ideas immanent in nervous activity," The Bulletin of Mathematical Biophysics, vol. 5, no. 4, pp. 115-133, 1943.

[2] J. Li, "New delay-dependent robust stability condition for neutral-type neural networks with mixed time delays," in Procedings of the 2nd International Conference on Industrial Mechatronics and Automation (ICIMA '10), vol. 1, pp. 486489, May 2010. 
[3] M. S. Mahmoud and A. Ismail, "Improved results on robust exponential stability criteria for neutral-type delayed neural networks," Applied Mathematics and Computation, vol. 217, no. 7, pp. 3011-3019, 2010.

[4] Y. Sun and J. Cao, "Stabilization of stochastic delayed neural networks with markovian switching," Asian Journal of Control, vol. 10, no. 3, pp. 327-340, 2008.

[5] J. F. Wang, J. G. Jian, and P. Yan, "Finite-time boundedness analysis of a class of neutral type neural networks with time delays," in Proceedings of the 6th International Symposium on Neural Networks on Advances in Neural Networks, vol. 1, pp. 395-404, 2009.

[6] J. Liu and G. Zong, "New delay-dependent asymptotic stability conditions concerning BAM neural networks of neutral type," Neurocomputing, vol. 72, no. 10-12, pp. 2549-2555, 2009.

[7] Q. Zhang, X. Wei, and J. Xu, "Global exponential stability for nonautonomous cellular neural networks with unbounded delays," Chaos, Solitons and Fractals, vol. 39, no. 3, pp. 11441151, 2009.

[8] H. Yang and T. Chu, "LMI conditions for stability of neural networks with distributed delays," Chaos, Solitons and Fractals, vol. 34, no. 2, pp. 557-563, 2007.

[9] B. Song, S. Xu, and Y. Zou, "Delay-dependent robust $H_{\infty}$ filtering for uncertain neutral stochastic time-delay systems," Circuits, Systems, and Signal Processing, vol. 28, no. 2, pp. 241256, 2009.

[10] Y. Li, S. Xu, B. Zhang, and Y. Chu, "Delay-dependent guaranteed cost control for uncertain neutral systems with distributed delays," International Journal of Control, Automation and Systems, vol. 6, no. 1, pp. 15-23, 2008.

[11] Y. Chen, W. X. Zheng, and A. Xue, "A new result on stability analysis for stochastic neutral systems," Automatica, vol. 46, no. 12, pp. 2100-2104, 2010.

[12] P. Balasubramaniam, A. Manivannan, and R. Rakkiyappan, "Exponential stability results for uncertain neutral systems with interval time-varying delays and Markovian jumping parameters," Applied Mathematics and Computation, vol. 216, no. 11, pp. 3396-3407, 2010.

[13] Y. Wang, Y. He, and X. Zhang, "Refined delay-dependent robust stability criteria of a class of uncertain mixed neutral and lur'e dynamical systems with interval time-varying," in Proceedings of the Chinese Control and Decision Conference (CCDC '11), pp. 4179-4184, Sichuan, China, May 2011.

[14] T. Yi, G. Liu, Y. Liu, and R. Wang, "Global robust stability analysis for uncertain stochastic neural networks of neutral-type with time-varying delays," in Proceedings of the International Conference on Electric Information and Control Engineering (ICEICE '11), pp. 1399-1403, April 2011.

[15] H. Chen, Y. Zhang, and P. Hu, "Novel delay-dependent robust stability criteria for neutral stochastic delayed neural networks," Neurocomputing, vol. 73, no. 13-15, pp. 2554-2561, 2010.

[16] W. Su and Y. Chen, "Global asymptotic stability analysis for neutral stochastic neural networks with time-varying delays," Communications in Nonlinear Science and Numerical Simulation, vol. 14, no. 4, pp. 1576-1581, 2009.

[17] L. Jin, "New LMI-based stability condition for neutral-type neural networks with variable and distributed delays," in Proceedings of the International Conference on Intelligent System Design and Engineering Application (ISDEA '10), pp. 10021005 , October 2010.

[18] J. E. Feng, S. Xu, and Y. Zou, "Delay-dependent stability of neutral type neural networks with distributed delays," Neurocomputing, vol. 72, no. 10-12, pp. 2576-2580, 2009.
[19] X. Li, "Global robust stability for stochastic interval neural networks with continuously distributed delays of neutral type," Applied Mathematics and Computation, vol. 215, no. 12, pp. 4370-4384, 2010.

[20] R. Rakkiyappan and P. Balasubramaniam, "New global exponential stability results for neutral type neural networks with distributed time delays," Neurocomputing, vol. 71, no. 4-6, pp. 1039-1045, 2008.

[21] R. Rakkiyappan and P. Balasubramaniam, "LMI conditions for global asymptotic stability results for neutral-type neural networks with distributed time delays," Applied Mathematics and Computation, vol. 204, no. 1, pp. 317-324, 2008.

[22] C. Y. Lu, "A delay-dependent approach to robust control for neutral uncertain neural networks with mixed interval timevarying delays," Nonlinearity, vol. 24, no. 4, pp. 1121-1136, 2011.

[23] K. Gu, "An integral inequality in the stability problem of timedelay systems," in Proceedings of the 39th IEEE Confernce on Decision and Control, pp. 2805-2810, December 2000.

[24] S. Boyd, L. El Ghaoui, E. Feron, and V. Balakrishnan, Linear Matrix Inequalities in System and Control Theory, vol. 15, SIAM Studies in Applied Mathematics, Philadelphia, Pa, USA, 1994.

[25] G. Hardy, J. E. Littlewood, and G. Pólya, Inequalities, Cambridge University Press, Cambridge, UK, 1988.

[26] V. B. Kolmanovski and A. D. Myshkis, Applied Theory of Functional Differential Equations, vol. 85, Springer, 1992.

[27] Y. He, M. Wu, G. P. Liu, and J. H. She, "Output feedback stabilization for a discrete-time system with a time-varying delay," IEEE Transactions on Automatic Control, vol. 53, no. 10, pp. 2372-2377, 2008.

[28] H. Gao, J. Lam, C. Wang, and Y. Wang, "Delay-dependent output-feedback stabilisation of discrete-time systems with time-varying state delay," IEE Proceedings Control Theory \& Applications, vol. 151, no. 6, pp. 691-698, 2004.

[29] L. El Ghaoui, F. Oustry, and M. AitRami, "A cone complementarity linearization algorithm for static output-feedback and related problems," IEEE Transactions on Automatic Control, vol. 42, no. 8, pp. 1171-1176, 1997.

[30] A. Bellen, N. Guglielmi, and A. E. Ruehli, "Methods for linear systems of circuit delay differential equations of neutral type," IEEE Transactions on Circuits and Systems I, vol. 46, no. 1, pp. 212-215, 1999.

[31] R. Rakkiyappan, P. Balasubramaniam, and R. Krishnasamy, "Delay-dependent stability of neutral systems with timevarying delays using delay-decomposition approach," Applied Mathematical Modelling, vol. 36, no. 5, pp. 2253-2261, 2012.

[32] M. N. A. Parlakçi, "Extensively augmented Lyapunov functional approach for the stability of neutral time-delay systems," IET Control Theory and Applications, vol. 2, no. 5, pp. 431-436, 2008.

[33] M. N. A. Parlakçi, "Robust stability of uncertain neutral systems: a novel augmented Lyapunov functional approach," IET Control Theory and Applications, vol. 1, no. 3, pp. 802-809, 2007. 

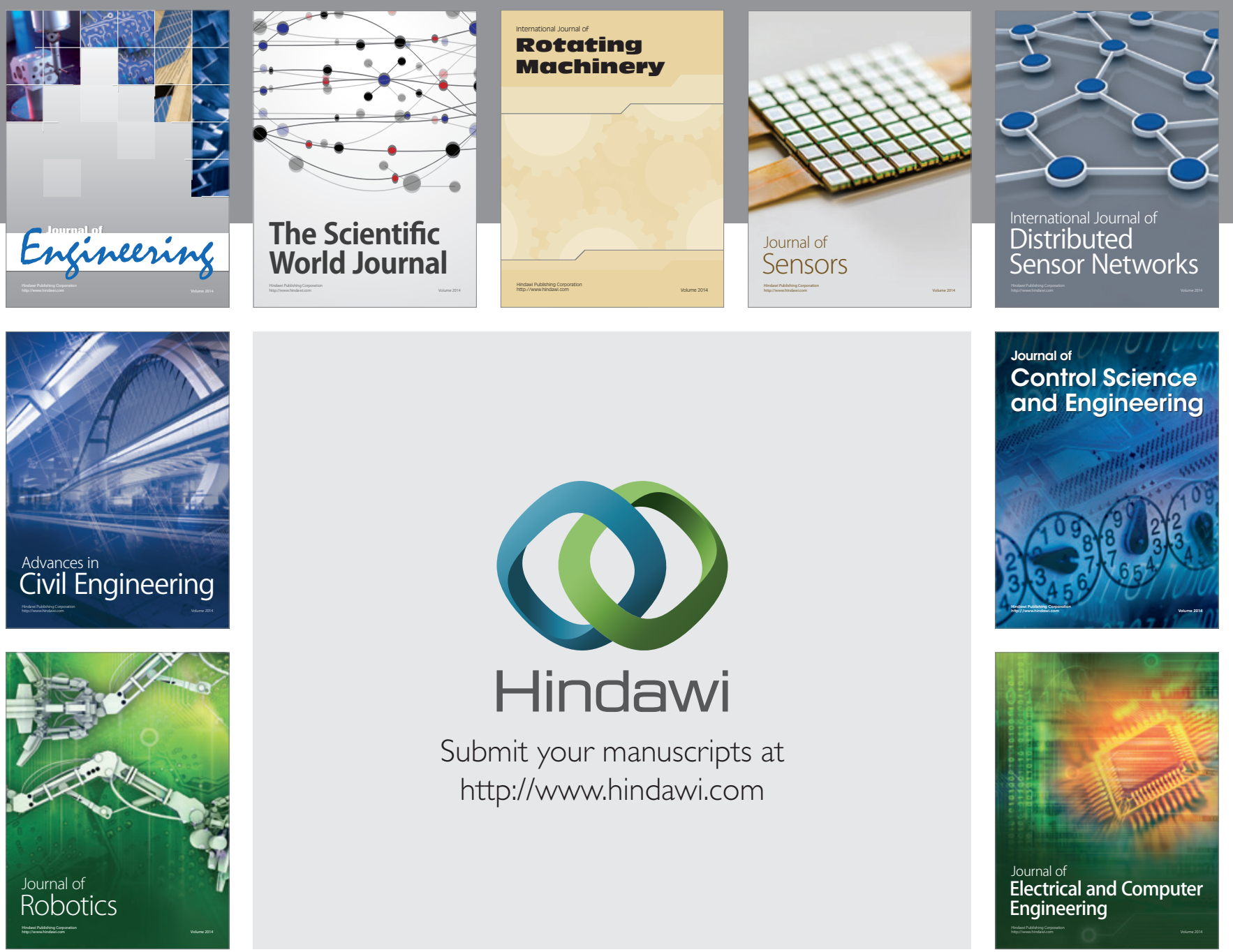

Submit your manuscripts at

http://www.hindawi.com
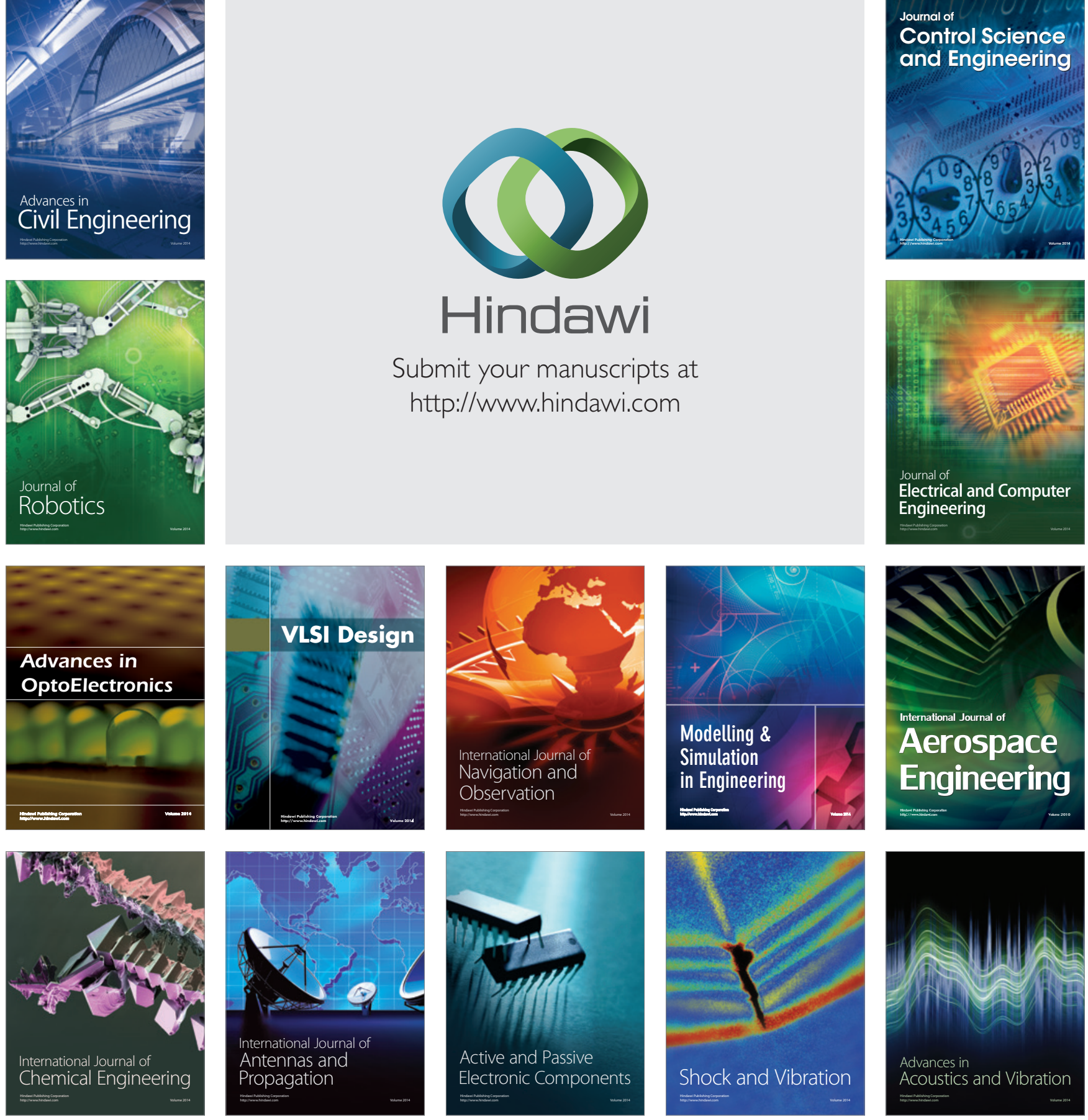